PROCEEDINGS OF THE

AMERICAN MATHEMATICAL SOCIETY

Volume 133, Number 6, Pages 1647-1656

S 0002-9939(04)07876-1

Article electronically published on December 21, 2004

\title{
ON THE BEHAVIOR OF WEAK CONVERGENCE UNDER NONLINEARITIES AND APPLICATIONS
}

\author{
DIEGO R. MOREIRA AND EDUARDO V. TEIXEIRA
}

(Communicated by Jonathan M. Borwein)

\begin{abstract}
This paper provides a sufficient condition to guarantee the stability of weak limits under nonlinear operators acting on vector-valued Lebesgue spaces. This nonlinear framework places the weak convergence in perspective. Such an approach allows short and insightful proofs of important results in Functional Analysis such as: weak convergence in $L^{\infty}$ implies strong convergence in $L^{p}$ for all $1 \leq p<\infty$, weak convergence in $L^{1}$ vs. strong convergence in $L^{1}$ and the Brezis-Lieb theorem. The final goal is to use this framework as a strategy to grapple with a nonlinear weak spectral problem on $W^{1, p}$.
\end{abstract}

\section{INTRODUCTION}

The weak continuity of operators between Banach spaces is an important topic in the applications of Functional Analysis. One can often take advantage of the locally compact structure of function spaces endowed with the weak topology, providing a better understanding of the behavior of these operators. This issue becomes much more interesting in the nonlinear environment.

Among nonlinear operators, there is a distinguished class called Nemytskii operators. In a certain sense, we are concerned about when they preserve the weak convergence. More precisely, we consider the notion of a.e. and weak convergence (a.e.w.), and formulate the problem $\alpha_{p, q}$ where $p, q \geq 1$ as follows: Let $f$ be a Carathéodory function and suppose that the Nemytskii operator associated to $f$ maps $L^{p}(\Omega, \mathbb{E})$ into $L^{q}(\Omega, \mathbb{F})$. Does $N_{f}$ map a.e.w. convergent sequences into weakly convergent sequences? Our purpose in this paper is to study under what conditions the problem $\alpha_{p, q}$ is affirmatively answered, and then apply the answer to analyze some linear and nonlinear problems. It is worthwhile to point out that our approach is developed for vector-valued function spaces, which has turned out to be not only a suitable tool to clarify important linear phenomena, but also an interesting technique to approach nonlinear problems.

Our paper is organized as follows. In section 2 we treat the problem $\alpha_{p, q}$ for $q>1$. Theorem 2.6 answers the question. The central idea of Theorem [2.6 was motivated by the real-valued version of the problem. However the vector-valued case is supported by nonstandard results in Functional Analysis and Vector Measure Theory. Afterwards, we illustrate how to use Theorem [2.6] to provide short proofs

Received by the editors April 24, 2003.

2000 Mathematics Subject Classification. Primary 46B03, 46B10, 46B20.

The second author is grateful for the financial support by CNPq - Brazil.

(C)2004 American Mathematical Society 
of some important classical results. Namely, we prove that weak convergence in $L^{\infty}$ implies strong convergence in $L^{p}$ for all $1 \leq p<\infty$ and a.e.w. convergence in $L^{p}$ implies strong convergence in $L^{q}$ for every $1 \leq q<p$. In section 3 we offer a new approach to the Brezis-Lieb theorem using the ideas developed in the previous section. Current proofs may not provide much insight into the underlying machinery. We hope that our proof remedies this. Section 4 deals with the problem $\alpha_{1,1}$. The strategy used here is quite different from section 2. As a consequence we obtain an extension of a result regarding the relationship between weak and strong convergence in $L^{1}$. Section 5 concerns an application of the ideas studied in section 2, namely, the weak convergence under nonlinearities, to treat a nonlinear weak spectral problem on $W^{1, p}(\Omega)$. More specifically, we study the solvability in $W^{1, p}(\Omega)$ of the following nonlinear functional equation:

$$
f(x, u(x))-\lambda u(x)=\psi(x),
$$

where $\lambda \in \mathbb{R}$ and $\psi \in W^{1, p}(\Omega)$. The ideas in this section illustrate how topological methods can be used to get regularity results for nonlinear functional equations, skipping the usual machinery often involved in such problems.

We believe the ideas contained in this paper may be applied to study a wide class of functional and partial differential equations involving many different types of nonlinearities.

\section{WeAK CONVERGENCE UNDER Nemytski nONLINEARIties}

Definition 2.1. Let $(\Omega, \mathcal{A}, \mu)$ be a measure space. Let $\mathbb{E}$ and $\mathbb{F}$ be separable Banach spaces. A function $f: \Omega \times \mathbb{E} \rightarrow \mathbb{F}$ is said to be a Carathéodory function if:

(a) for each fixed $v \in \mathbb{E}$ the function $x \mapsto f(x, v)$ is $\mathcal{A}$-measurable;

(b) for $\mu$-almost everywhere fixed $x \in \Omega$ the function $v \mapsto f(x, v)$ is continuous.

We write $f \in(C)$ to indicate that $f$ is a Carathéodory function. In this case, the map $x \mapsto f(x, u(x))$ is $\mathcal{A}$-measurable whenever $u$ is an $\mathbb{E}$-valued $\mathcal{A}$-measurable function and will be denoted by $N_{f}(u)$. The correspondence $u \mapsto N_{f}(u)$ is called the Nemytskii operator. This is of special interest when $N_{f}$ acts on Lebesgue spaces. The next result characterizes these cases.

Theorem 2.2 (Lucchetti-Patrone). Let $\mathbb{E}, \mathbb{F}$ be separable Banach spaces. The Nemytskii operator $N_{f}$ maps $L^{p}(\Omega, \mathbb{E})$ into $L^{q}(\Omega, \mathbb{F}), 1 \leq p, q<+\infty$, if and only if there exist a constant $a>0$ and a function $b(x) \in L_{+}^{q}(\Omega)$ such that

$$
\|f(x, v)\|_{\mathbb{F}} \leq a \cdot\|v\|_{\mathbb{E}}^{p / q}+b(x) .
$$

In this case, the operator $N_{f}$ is continuous and bounded in the sense that it maps bounded sets into bounded sets.

The first natural question which arises here is the weak sequential continuity of the Nemytskii operators acting on $L^{p}$ spaces. The next example shows that there is no hope for such a result to be true.

Example 2.3. Let $\Omega=\left(0, \frac{\pi}{2}\right)$ and $u_{n}(x)=\sin (n x)$. By Riemann-Lebesgue's lemma, $u_{n} \rightarrow 0$ in $L^{2}\left(0, \frac{\pi}{2}\right)$. Let $f:\left(0, \frac{\pi}{2}\right) \times \mathbb{R} \rightarrow \mathbb{R}$ be given by $f(x, t)=t^{+}$. Now we note that $\left\langle 1, N_{f}\left(u_{n}\right)\right\rangle_{L^{2}}=\frac{1}{n} \int_{0}^{n \frac{\pi}{2}}(\sin (y))^{+} d y$. Then

$$
\limsup _{n}\left\langle 1, N_{f}\left(u_{n}\right)\right\rangle_{L^{2}} \geq \lim _{s} \frac{1}{4 s+2} \sum_{k=0}^{s} \int_{2 k \pi}^{(2 k+1) \pi} \sin y d y=\frac{1}{2} .
$$


The main point in Example 2.3 is the oscillation of the sequence $u_{n}$. In order to focus the problem on the right way we have to introduce the following concept.

Definition 2.4. Let $(X, \mathcal{A}, \mu)$ be a measure space and $\mathcal{F}(X, \mathbb{E})$ a topological vector space of $\mathbb{E}$-valued functions defined on $X$. We write $f_{n} \rightarrow f$ a.e.w. (almost everywhere and weakly) in $\mathcal{F}(X, \mathbb{E})$ if $f_{n} \rightarrow f \mu$ - a.e. in $X$ and $f_{n} \rightarrow f$ in $\mathcal{F}(X, \mathbb{E})$.

The most frequent source of examples of a.e.w. convergence comes from boundedness in the Sobolev spaces $W^{m, p}(\Omega)$ for $m \geq 1$ and $1 \leq p \leq \infty$. Using the previous definition we can restate our question as: Is the a.e.w. convergence preserved by Nemytskii operators? Stating it in a precise way, our problem is:

$\left(\alpha_{p, q}\right)\left\{\begin{array}{c}f \in(C) \\ N_{f} \text { maps } L^{p}(\Omega, \mathbb{E}) \text { into } L^{q}(\Omega, \mathbb{F}) \\ \text { Does } N_{f} \text { map a.e.w. convergent sequences into weakly convergent sequences? }\end{array}\right.$

The rest of this section will be devoted to studying the problem $\alpha_{p, q}$ and some consequences. The problem $\alpha_{1,1}$ has turned out to be more delicate and it will be fully studied in section 4 . The next example deals with the question $\alpha_{p, 1}$, for $p>1$.

Example 2.5. Let $1<p<\infty$. The answer to the problem $\alpha_{p, 1}$ is negative. Indeed let $\Omega$ be a domain in $\mathbb{R}^{N}$. Without losing generality, let us suppose $0 \in \Omega$. Set $f: \Omega \times \mathbb{R} \rightarrow \mathbb{F}, f(x, t)=|t|^{p} \cdot \nu_{0}$, where $\nu_{0} \in \mathbb{F} \backslash\{0\}$. The Nemytskii operator $N_{f}$ maps $L^{p}(\Omega)$ into $L^{1}(\Omega, \mathbb{F})$. Set $u_{n} \in L^{p}(\Omega), u_{n}=\left|B_{n}\right|^{-1 / p} \chi_{B_{n}}$, where $B_{n}=\left\{x \in \mathbb{R}^{N} ;|x|<1 / n\right\}$. Since $u_{n} \rightarrow 0$ a.e. in $\Omega$ and $\left\|u_{n}\right\|_{p}=1$, by reflexivity, we may assume $u_{n} \rightarrow 0$ a.e.w. Our claim is that $N_{f}\left(u_{n}\right) \dashv 0$ in $L^{1}(\Omega, \mathbb{F})$. In fact, from the Hahn-Banach theorem, there exists a $\psi \in \mathbb{F}^{*}$ such that $\psi\left(\nu_{0}\right)=1$. Define $\Psi \in\left[L^{1}(\Omega, \mathbb{F})\right]^{*}$ by

$$
\Psi(\xi)=\int_{\Omega} \psi \circ \xi(x) d x
$$

We obtain

$$
1=\left|B_{n}\right|^{-1} \int_{\Omega} \chi_{B_{n}}(x) d x=\int_{\Omega} \psi\left(N_{f}\left(u_{n}\right)\right)(x) d x=\Psi\left(N_{f}\left(u_{n}\right)\right) \not \rightarrow 0 .
$$

Theorem 2.6. Let $1 \leq p<+\infty, 1<q<\infty$ and $(\Omega, \mathcal{A}, \mu)$ be a $\sigma$-finite measure space. If $\mathbb{F}$ is reflexive, then the answer of problem $\alpha_{p, q}$ is affirmative.

Proof. Indeed, let us suppose $u_{n} \rightarrow u$ a.e.w. in $L^{p}(\Omega, \mathbb{E})$. Since $\left(u_{n}\right)$ is bounded in $L^{p}(\Omega, \mathbb{E})$, by Theorem 2.2 $\left(N_{f}\left(u_{n}\right)\right)$ is bounded in $L^{q}(\Omega, \mathbb{F})$. Once $\mathbb{F}$ is reflexive, so is its dual. By the Phillips theorem, $\mathbb{F}^{*}$ has the Radon-Nikodým property. Thus, $\left[L^{q}(\Omega, \mathbb{F})\right]^{*}=L^{q^{\prime}}\left(\Omega, \mathbb{F}^{*}\right)$. This implies that $L^{q}(\Omega, \mathbb{F})$ is reflexive. Hence, taking a subsequence if necessary, we may assume that $N_{f}\left(u_{m}\right) \rightarrow v \in L^{q}(\Omega, \mathbb{F})$. Clearly, $N_{f}\left(u_{m}\right) \rightarrow N_{f}(u)$ a.e. in $\Omega$. By hypothesis, there exists a sequence $\left\{\Omega_{j}\right\}_{j=1}^{\infty}$ of measurable subsets of $\Omega$ such that:

- $\mu\left(\Omega_{j}\right)<+\infty$ for each $j \geq 1$,

- $\Omega=\bigcup_{j=1}^{\infty} \Omega_{j}$.

Let $j \geq 1$ be fixed. From Egorov's theorem, given $\varepsilon>0$ there is a subset $A_{\varepsilon}$ of $\Omega_{j}$ with $\mu\left(A_{\varepsilon}\right)<\varepsilon$ such that $N_{f}\left(u_{n}\right) \rightarrow N_{f}(u)$ in $L^{\infty}\left(\Omega_{j} \backslash A_{\varepsilon}, \mathbb{F}\right)$. In particular this convergence takes place in $L^{p}\left(\Omega_{j} \backslash A_{\varepsilon}, \mathbb{F}\right)$. It follows that $N_{f}(u)=v$ a.e. in 
$\Omega_{j} \backslash A_{\varepsilon}$. Therefore, the set $A_{j}=\left\{x \in \Omega_{j}: N_{f}(u)(x) \neq v(x)\right\}$ has measure zero once $A_{j} \subset A_{\varepsilon}$, for every $\varepsilon>0$. To finish, setting $A=\bigcup_{j=1}^{\infty} A_{j}$, we have that $A=\left\{x \in \Omega: N_{f}(u)(x) \neq v(x)\right\}$. In this way, $\mu(A)=0$ and the result follows.

In the next result as well as in the last section we shall make use of the theory of Sobolev spaces. For the convenience of the readers, we provide the following definition.

Definition 2.7. Let $\Omega \subset \mathbb{R}^{N}$ be an open set and $1 \leq p \leq \infty$. The Sobolev space $W^{1, p}(\Omega)$ is defined to be:

$$
W^{1, p}(\Omega):=\left\{u \in L^{p}(\Omega) \mid \begin{array}{c}
\exists g_{1}, g_{2}, \ldots, g_{N} \in L^{p}(\Omega) \text { such that } \\
\int_{\Omega} u \frac{\partial \varphi}{\partial x_{i}}=-\int_{\Omega} g_{i} \varphi \forall \varphi \in C_{c}^{\infty}(\Omega) \forall i=1,2, \ldots, N
\end{array}\right\} .
$$

Such $g_{i}$ 's on the above definition are unique (a.e.). This allows us to denote $g_{i}=\partial_{i} u$ and call it the $i^{\text {th }}$ weak derivative of $u$. We should also mention that the Sobolev spaces $W^{1, p}$ inherit the same functional properties of the $L^{p}$-spaces. For further details see, for instance, [1] or [2].

Proposition 2.8. Let $(\Omega, \mathcal{A}, \mu)$ be a finite measure space. If $u_{n} \rightarrow u$ a.e.w. in $L^{p}(\Omega, \mathbb{E})$ with $p>1$, then $u_{n} \rightarrow u$ in $L^{q}(\Omega, \mathbb{E})$ for all $1 \leq q<p$. In particular, if $\Omega$ is an open subset of $\mathbb{R}^{N}, W^{1, p}(\Omega)$ is compactly embedded in $L^{q}(\Omega)$, for all $1 \leq q<p$, without any regularity condition on $\partial \Omega$.

Proof. Let us fix $0<\varepsilon<p-1$. Set $f: \mathbb{E} \rightarrow \mathbb{R}$ given by $f(\nu)=\|\nu\|_{\mathbb{E}}^{p-\varepsilon}$. $N_{f}$ maps $L^{p}(\Omega, \mathbb{E})$ into $L^{p / p-\varepsilon}(\Omega)$. From Theorem 2.6 $f\left(u_{n}\right) \rightarrow f(u)$ in $L^{p / p-\varepsilon}(\Omega)$. In particular

$$
\int_{\Omega}\left\|u_{n}\right\|_{\mathbb{E}}^{p-\varepsilon} d x \rightarrow \int_{\Omega}\|u\|_{\mathbb{E}}^{p-\varepsilon} d x .
$$

Thus, $u_{n} \rightarrow u$ a.e. and $\left\|u_{n}\right\|_{L^{p-\varepsilon}(\Omega, \mathbb{E})} \rightarrow\|u\|_{L^{p-\varepsilon}(\Omega, \mathbb{E})}$. This implies that $u_{n} \rightarrow u$ in $L^{p-\varepsilon}(\Omega, \mathbb{E})$. Moreover, since $L^{p-\varepsilon}(\Omega, \mathbb{E}) \hookrightarrow L^{r}(\Omega, \mathbb{E})$ for $1 \leq r \leq p-\varepsilon$, we conclude that $u_{n} \rightarrow u$ in $L^{q}(\Omega, \mathbb{E})$ for all $q \in[1, p-\varepsilon]$. Letting $\varepsilon \rightarrow 0$, we get the result. The second part of the proposition follows from the first part with the additional fact that every bounded sequence in $W^{1, p}(\Omega)$ has a subsequence that converges a.e.w. in $W^{1, p}(\Omega)$.

Proposition 2.9. Let $\Omega$ be an open bounded domain in $\mathbb{R}^{N}$. If a sequence $u_{n} \rightarrow u_{0}$ in $L^{\infty}(\Omega)$, then $u_{n} \rightarrow u_{0}$ strongly in every $L^{p}(\Omega)$, for $1 \leq p<\infty$.

Proof. For each $x \in \Omega$, let us consider the functionals $\varphi_{r}^{x}: L^{\infty}(\Omega) \rightarrow \mathbb{R}$ defined by

$$
\varphi_{r}^{x}(f):=\frac{1}{\left|B_{r}(x)\right|} \int_{B_{r}(x)} f(\xi) d \xi
$$

$\varphi_{r}^{x}$ are linear functionals and $\left\|\varphi_{r}^{x}\right\|_{L^{\infty}(\Omega)^{*}}=1$. Therefore, passing to a subnet if necessary, we may assume that $\varphi_{r}^{x} \stackrel{*}{\rightarrow} \varphi^{x}$, as $r \rightarrow 0$. From Lebesgue's differentiation theorem, for every $f \in L^{\infty}(\Omega)$, there exists a set $A_{f}$, with $\left|A_{f}\right|=0$, such that $\varphi_{r}^{x}(f) \stackrel{r \rightarrow 0}{\longrightarrow} f(x)$ in $\Omega \backslash A_{f}$. Let $A=\bigcup_{n=0}^{\infty} A_{u_{n}}$. In this way $|A|=0$, and for every 
$x \in \Omega \backslash A$ it follows that

$$
\varphi_{r}^{x}\left(u_{n}\right) \stackrel{r \rightarrow 0}{\longrightarrow} u_{n}(x)=\varphi^{x}\left(u_{n}\right) \stackrel{n \rightarrow \infty}{\longrightarrow} \varphi^{x}\left(u_{0}\right)=u_{0}(x) .
$$

Thus, $u_{n} \rightarrow u_{0}$ a.e. in $\Omega$. Given $p \in[1, \infty)$, from the continuous embedding $L^{\infty}(\Omega) \hookrightarrow L^{p+1}(\Omega)$ we get $u_{n} \rightarrow u_{0}$ a.e.w. in $L^{p+1}(\Omega)$, and by Proposition [2.8 $u_{n} \rightarrow u_{0}$ in $L^{p}(\Omega)$.

Remark 2.10. (i) This follows from the proof that one just needs to verify in the hypothesis of Theorem 2.6 the boundedness of $u_{n}$ in $L^{p}(\Omega, \mathbb{E})$, instead of weak convergence of it. When $\mathbb{E}$ is not reflexive, this assumption is much easier to check.

(ii) It is worthwhile to point out that Theorem 2.6 holds for any continuous (nonlinear) bounded operator $\Phi: L^{p}(\Omega, \mathbb{E}) \rightarrow L^{q}(\Omega, \mathbb{F})$ provided $\Phi\left(\chi_{A} f\right)=\chi_{A} \Phi(f)$ holds for every finite measurable subset of $\Omega$. The proof of this fact follows from a small variant of the proof of Theorem 2.6.

(iii) Proposition 2.8 is sharp since $W^{1, p}(\Omega)$ may not be compactly embedded into $L^{q}(\Omega)$, for $q \geq p$. This fact can be found in 1$]$.

(iv) Proposition 2.9 was motivated by 9 which brings a different and abstract approach. The last step in the proof of Proposition 2.9 follows also from the Lebesgue dominated convergence theorem.

\section{A new approach to the Brezis-Lieb theorem}

In [3], H. Brezis and E. Lieb provided a powerful result concerning a refinement of Fatou's lemma which has been shown to be very useful in a wide class of problems.

Theorem 3.1 (Brezis-Lieb). Let $0<p<\infty$. Suppose $u_{n} \rightarrow u$ a.e. and $\left\|u_{n}\right\|_{L^{p}(\Omega)}$ $\leq C<\infty$. Then

$$
\lim _{n \rightarrow \infty}\left(\left\|u_{n}\right\|_{L^{p}(\Omega)}^{p}-\left\|u_{n}-u\right\|_{L^{p}(\Omega)}^{p}\right)=\|u\|_{L^{p}(\Omega)}^{p} .
$$

Our purpose in this section is to provide an alternative proof of Theorem 3.1 which might give insight as to why this type of phenomenon should hold. Besides this, our approach may be used to get useful extensions on this matter. Indeed, let us, for the moment, work on the case where $p=2 m$ is an even number. In this case, we can develop the expression above by Newton's binomial expansion and get

$$
\begin{aligned}
& \int_{\Omega}\left|u_{n}\right|^{2 m}-\left|u_{n}-u\right|^{2 m}=\int_{\Omega}-\sum_{i=1}^{2 m}\left(\begin{array}{c}
2 m \\
i
\end{array}\right) u_{n}^{2 m-i} \cdot(-1)^{i} u^{i} \stackrel{n \rightarrow \infty}{\longrightarrow} \int_{\Omega}|u|^{2 m} \text { as } n \rightarrow \infty, \\
& \text { since } \sum_{i=1}^{2 m}\left(\begin{array}{c}
2 m \\
i
\end{array}\right)(-1)^{i}=-1 \text { and from Theorem 2.6 } u_{n}^{2 m-i} \rightarrow u^{2 m-i} \text { in } L^{i^{\prime}}(\Omega) .
\end{aligned}
$$

This gives us a better understanding as to what is really behind this issue. In fact, the key point of the computations above is that the highest power in Newton's binomial expansion cancels out, avoiding the interference of the $\alpha_{2 m, 1}$ problem. For the remaining powers, Theorem 2.6 can be applied.

To treat the general case we need a lemma regarding an inequality on the real line. A similar inequality is also needed in the original proof.

Lemma 3.2. Let $1<p<\infty$. Given $\varepsilon>0$, there exist a constant $C_{\varepsilon}>0$ and a function $\delta(\varepsilon) \rightarrow 0$ as $\varepsilon \rightarrow 0$ such that

$$
\left.|| a\right|^{p}-|a-b|^{p}-|b|^{p}\left|\leq C_{\varepsilon}\right| b|\cdot| a-\left.b\right|^{p-1}+\delta(\varepsilon)|b|^{p} \quad \forall a, b \in \mathbb{R} .
$$


Proof. Let us consider the function $\varphi: \mathbb{R} \backslash\{1\} \rightarrow \mathbb{R}$ given by

$$
\varphi(x)=\frac{\left.|| x\right|^{p}-|x-1|^{p}-1 \mid}{|x-1|^{p-1}} .
$$

Although $\varphi$ blows up at 1 , we have $\lim _{|x| \rightarrow \infty} \varphi(x)=p$. So given $\varepsilon>0$, there exists a constant $C_{\varepsilon}$ such that $\left.|| x\right|^{p}-|x-1|^{p}-1\left|\leq C_{\varepsilon}\right| x-\left.1\right|^{p-1}$ whenever $|x-1|>\varepsilon$. On the other hand, if $|x-1| \leq \varepsilon$, we have

$$
\left.|| x\right|^{p}-|x-1|^{p}-1 \mid \leq \varepsilon^{p}+\tilde{\delta}(\varepsilon), \quad \text { where } \tilde{\delta}(\varepsilon)=\left.\sup _{|t-1| \leq \varepsilon}|| t\right|^{p}-1 \mid .
$$

Adding the above two inequalities we get that

$$
\left.|| x\right|^{p}-|x-1|^{p}-1\left|\leq C_{\varepsilon}\right| x-\left.1\right|^{p-1}+\varepsilon^{p}+\tilde{\delta}(\varepsilon),
$$

for every $x \in \mathbb{R}$. Finally, given two nonzero real numbers $a$ and $b$, we make $x=\frac{a}{b}$ in the above inequality and the result follows.

Proof of the Brezis-Lieb theorem using Theorem 2.6. As pointed out in [3], for $0<$ $p \leq 1$ the theorem follows immediately. Let us deal with $1<p<\infty$. From the last lemma, we have

$$
\left.\int_{\Omega}|| u_{n}\right|^{p}-\left|u_{n}-u\right|^{p}-|u|^{p}\left|d x \leq C_{\varepsilon} \int_{\Omega}\right| u|\cdot| u_{n}-\left.u\right|^{p-1} d x+\delta(\varepsilon) \int_{\Omega}|u(x)|^{p} d x .
$$

Consider the map $f: \Omega \times \mathbb{R} \rightarrow \mathbb{R}$ defined by $f(x, t)=|t-u(x)|^{p-1}$. $N_{f}$ maps $L^{p}(\Omega)$ into $L^{\frac{p}{p-1}}(\Omega)$ and therefore, from Theorem [2.6, $N_{f}\left(u_{n}\right) \rightarrow 0$ in $L^{\frac{p}{p-1}}(\Omega)$. Letting $n \rightarrow \infty$ in (3.1) and after letting $\varepsilon \rightarrow 0$, we conclude the proof of Theorem 3.1

Remark 3.3. In a more general sense, suppose $j: \mathbb{E} \rightarrow \mathbb{F}$ is a map such that

$$
\|j(u)-j(u-v)-j(v)\|_{\mathbb{F}} \leq C_{\varepsilon}\|v\|_{\mathbb{E}}^{\mu(\varepsilon)} \cdot\|u-v\|_{\mathbb{E}}^{p-\mu(\varepsilon)}+\delta(\varepsilon)\left(\|v\|_{\mathbb{E}}^{p}+\|u\|_{\mathbb{E}}^{p}\right),
$$

where $\mu(\varepsilon)>0$, and $\delta(\varepsilon)$ is a modulus of continuity. Then it follows that

$$
\int_{\Omega}\left\|j\left(u_{n}\right)-j\left(u_{n}-u\right)-j(u)\right\|_{\mathbb{F}} \longrightarrow 0
$$

as $n \rightarrow \infty$, whenever $u_{n} \rightarrow u$ a.e. and $\left\|u_{n}\right\|_{L^{p}(\Omega, \mathbb{E})} \leq C<\infty$.

\section{The Problem $\alpha_{1,1}$}

In this section, we bring our attention to the problem $\alpha_{1,1}$. To this end we need a general version of the Dunford-Pettis theorem.

Theorem 4.1 (Talagrand). Let $\mathbb{F}$ be a weakly sequentially complete Banach space. Let $\mathcal{F} \subset L^{1}(\Omega, \mathbb{F})$ be a bounded convex subset. Then $\mathcal{F}$ is weakly relatively compact if and only if it satisfies the following two conditions:

(1) $\|\mathcal{F}\|_{\mathbb{F}}:=\left\{\|\varphi\|_{\mathbb{F}}: \Omega \rightarrow \mathbb{R}: \varphi \in \mathcal{F}\right\} \subset L^{1}(\Omega)$ is weakly relatively compact;

(2) for each sequence $\left(\varphi_{n}\right)$ in $\mathcal{F}$, the set of $x \in \Omega$ such that there is a $k$ for which the sequence $\left(\varphi_{n}\right)_{n \geq k}$ is equivalent to the vector basis of $\ell_{1}$ has measure zero.

Let us point out that condition (1) above is equivalent to the countable additivity of the integrals $\int_{\Omega}\|\varphi\|_{\mathbb{F}} d \mu$ being uniform with respect to $\|\varphi\|_{\mathbb{F}}$ (see [5]). 
Theorem 4.2. If $\mathbb{E}$ is weakly sequentially complete and $\mathbb{F}$ is reflexive, then the answer to the problem $\alpha_{1,1}$ is affirmative.

Proof. Let $u_{n} \rightarrow u$ a.e.w. in $L^{1}(\Omega, \mathbb{E})$. Defining $u_{0}=u$, the set $K=\left\{u_{n}: n \geq 0\right\}$ is weakly compact in $L^{1}(\Omega, \mathbb{E})$, by the Eberlein-Smulian theorem. Let us denote $X=\overline{c o}(K)$. From the Krein-Smulian theorem, $X$ is weakly compact; thus, in particular, Theorem 4.1 says that $\|X\|_{\mathbb{E}}$ is uniformly countably additive. This means that for each decreasing sequence $\left\{\Omega_{j}\right\}$ with void intersection, the limit

$$
\lim _{j \rightarrow \infty} \int_{\Omega_{j}}\|\varsigma\|_{\mathbb{E}} d \mu=0
$$

is uniform with respect to $\varsigma$ in $X$. By Theorem 2.2 the Carathéodory function $f$ satisfies the following growth condition:

$$
\|f(x, v)\|_{\mathbb{F}} \leq a\|v\|_{\mathbb{E}}+b(x)
$$

where $a>0$ and $b(x) \in L_{+}^{1}(\Omega)$. Let $Y=\overline{c o}\left(N_{f}(K)\right)$. If $v \in c o\left(N_{f}(K)\right)$, there exist functions $u_{k_{1}}, \ldots, u_{k_{n}} \in K$ and positive numbers $\lambda_{1}, \ldots, \lambda_{n}$ fulfilling $\sum_{i=1}^{n} \lambda_{i}=1$ such that $v=\sum_{i=1}^{n} \lambda_{i} \cdot N_{f}\left(u_{k_{i}}\right)$.

In this way

$$
\int_{\Omega_{j}}\|v(x)\|_{\mathbb{F}} d \mu=\int_{\Omega_{j}}\left\|\sum_{i=1}^{n} \lambda_{i} \cdot N_{f}\left(u_{k_{i}}\right)(x)\right\|_{\mathbb{F}} d \mu \leq \int_{\Omega_{j}} \sum_{i=1}^{n} \lambda_{i}\left\|N_{f}\left(u_{k_{i}}\right)(x)\right\|_{\mathbb{F}} d \mu
$$

and

$$
\begin{aligned}
\int_{\Omega_{j}} \sum_{i=1}^{n} \lambda_{i}\left\|N_{f}\left(u_{k_{i}}\right)(x)\right\|_{\mathbb{F}} d \mu & \leq a \cdot \int_{\Omega_{j}} \sum_{i=1}^{n} \lambda_{i} \cdot\left\|u_{k_{i}}(x)\right\|_{\mathbb{E}} d \mu+\int_{\Omega_{j}} b(x) d \mu \\
& \rightarrow 0 \text { uniformly as } j \rightarrow \infty .
\end{aligned}
$$

We have just verified condition (1) of Theorem 4.1 for $Y$. By hypothesis, we get condition (2) for free, because $\mathbb{F}$ does not contain a copy of $\ell_{1}$ since it is reflexive. Therefore by Theorem 4.1 the set $Y$ is weakly compact, and thus so is ${\overline{N_{f}(K)}}^{w}$. Using again the Eberlein-Smulian theorem, up to a subsequence we may assume $N_{f}\left(u_{n}\right) \rightarrow v$ in $L^{1}(\Omega, \mathbb{F})$. Since $N_{f}\left(u_{n}\right) \rightarrow N_{f}(u)$ a.e. in $\Omega$, we conclude, as done in the final step of Theorem [2.6. that $v=N_{f}(u)$ and then $N_{f}\left(u_{n}\right) \rightarrow N_{f}(u)$ in $L^{1}(\Omega, \mathbb{F})$.

It is interesting to notice that we could change a.e. convergence to convergence in measure in every subset of finite measure in the hypothesis of Theorem 2.6 as well as in the hypothesis of Theorem 4.2. In this way, we cannot only generalize to vector-valued functions, but also give a straightforward proof of a remarkable theorem concerning weak convergence vs. strong convergence in $L^{1}$.

Theorem 4.3. Let $\mathbb{E}$ be a weakly sequentially complete Banach space, and let $u_{n} \rightarrow$ $u$ in $L^{1}(\Omega, \mathbb{E})$. Then $\left\{u_{n}\right\}$ converges strongly to $u$ if and only if $\left\{u_{n}\right\}$ converges in measure to $u$ in every subset of finite measure. 
Proof. Since $u_{n}-u \rightarrow 0$ in $L^{1}(\Omega, \mathbb{E})$ and, by hypothesis, $u_{n}$ converges to $u$ in measure in every subset of finite measure, it follows from Theorem 4.2 that $N_{\|\cdot\|_{\mathbb{E}}}\left(u_{n}-u\right) \rightarrow N_{\|\cdot\|_{\mathbb{E}}}(0)=0$ in $L^{1}(\Omega)$. In particular

$$
\lim _{n \rightarrow \infty} \int_{\Omega}\left\|u_{n}-u\right\|_{\mathbb{E}}=0 .
$$

Remark 4.4. Theorem 4.3 gives the reason for the negative answer to the problem $\alpha_{p, 1}$ for $p>1$. In fact, the problem $\alpha_{p, 1}$ is equivalent to its strong version:

$\alpha_{p, 1}$-strong version: $u_{n} \rightarrow u$ a.e.w. in the $L^{p} \Longrightarrow N_{f}\left(u_{n}\right) \rightarrow N_{f}(u)$ in the $L^{1}$-norm.

\section{A nonlinear WEAK SPECTRAL PROBlem on $W^{1, p}(\Omega)$}

We shall provide an application of Theorem 2.6 by solving a general nonlinear equation on the Sobolev spaces $W^{1, p}(\Omega)$. The problem studied here is a very natural question for the Nemytskii operator on Sobolev spaces. Indeed, the problem we shall work on is:

Let $1<p<\infty$ and $\Omega$ be a domain in $\mathbb{R}^{N}$. Let $f: \Omega \times \mathbb{R} \rightarrow \mathbb{R}$ be such that

(1) For a.e. $x \in \Omega, f(x, \cdot)$ is a Lipschitzian function and $\sup _{x \in \Omega}\|f(x, \cdot)\|_{\text {Lip }}=L$ is finite.

(2) $f(\cdot, t) \in W^{1, p}(\Omega)$, and $\|f(\cdot, t)\|_{W^{1, p}} \leq C, \forall t \in \mathbb{R}$.

Given a $\psi \in W^{1, p}(\Omega)$, and given a $\lambda \in \mathbb{R}$ we are interested in finding a $u \in W^{1, p}(\Omega)$ such that

$$
f(x, u(x))-\lambda u(x)=\psi(x) \text { a.e. } x \in \Omega .
$$

Theorem 5.1. Problem (5.1) is affirmatively answered for all $\lambda>L$. Moreover the solution is unique and the resolvent operator $\left(N_{f}-\lambda I d\right)^{-1}: W^{1, p}(\Omega) \rightarrow W^{1, p}(\Omega)$ is sequentially weakly continuous.

Proof. By changing $f$ by $f-f(x, 0)$, we may assume, without loss of generality, that $f(x, 0)=0$, for a.e. $x \in \Omega$. Let us start by estimating $\left\|N_{f}(\xi)\right\|_{W^{1, p}(\Omega)}$ :

$$
\begin{aligned}
\left\|N_{f}(\xi)\right\|_{W^{1, p}} & =\|f(x, \xi(x))\|_{L^{p}}+\sum_{j=1}^{N}\left\|\partial_{j} f(x, \xi(x))\right\|_{L^{p}} \\
& \leq L\|\xi\|_{L^{p}}+\sum_{j=1}^{N}\left\|\left(\partial_{j} f\right)(x, \xi(x))+\left(\partial_{t} f\right)(x, \xi(x)) \cdot \partial_{j} \xi(x)\right\|_{L^{p}} \\
& \leq L\|\xi\|_{W^{1, p}}+C .
\end{aligned}
$$

The inequality above tells us that $N_{f}: W^{1, p}(\Omega) \rightarrow W^{1, p}(\Omega)$ is a bounded operator. This fact added with that every weakly convergent sequence in $W^{1, p}(\Omega)$ has a subsequence converging a.e. implies that $N_{f}$ is sequentially weakly continuous. Indeed, it follows from the same kind of argument found in the final step of Theorem 2.6. Let us define $\Lambda: W^{1, p}(\Omega) \rightarrow W^{1, p}(\Omega)$ by

$$
\Lambda(\xi)=\frac{1}{\lambda}[f(x, \xi(x))-\psi] .
$$

We observe that once $N_{f}$ is sequentially weakly continuous, so is $\Lambda$. Moreover, the solvability of equation (5.1) is equivalent to finding a fixed point of $\Lambda$. For each 
$\xi \in W^{1, p}(\Omega)$, it follows that

$$
\begin{aligned}
\lambda^{-1}\|f(x, \xi(x))-\psi\|_{W^{1, p}} & \leq \lambda^{-1}\left(\|f(x, \xi(x))\|_{W^{1, p}}+\|\psi\|_{W^{1, p}}\right) \\
\leq & \lambda^{-1}\left(L\|\xi\|_{W^{1, p}}+C+\|\psi\|_{W^{1, p}}\right) .
\end{aligned}
$$

Let us fix $M>\frac{C+\|\psi\|_{W^{1, p}}}{\lambda-L}$. For such an $M$ we see that if $\|\xi\|_{W^{1, p}(\Omega)} \leq M$, then

$$
\|\Lambda(\xi)\|_{W^{1, p}} \leq \frac{L}{\lambda} \cdot M+\frac{C+\|\psi\|_{W^{1, p}}}{\lambda} \leq M
$$

In other words, $\Lambda$ maps the closed ball of radius $M$ in $W^{1, p}(\Omega)$ into itself. Let $X$ denote $\overline{B_{W^{1, p}}(M)}$ endowed with the weak topology. So $X$ is a compact and convex set of a locally convex space. In addition, as we pointed out before, $\Lambda: X \rightarrow X$ is a continuous map. Invoking the Leray-Schauder-Tychonoff fixed point theorem we conclude that $\Lambda$ has a fixed point which is precisely a solution to (5.1). We now turn our attention to uniqueness. Let us suppose that there exist $u_{1}, u_{2} \in W^{1, p}(\Omega)$ such that

$$
f\left(x, u_{1}(x)\right)-\lambda u_{1}(x)=\psi(x) \quad \text { and } \quad f\left(x, u_{2}(x)\right)-\lambda u_{2}(x)=\psi(x) .
$$

Subtracting these above equations, we find

$$
f\left(x, u_{1}(x)\right)-f\left(x, u_{2}(x)\right)=\lambda\left(u_{1}(x)-u_{2}(x)\right) .
$$

Therefore

$$
\left|f\left(x, u_{1}(x)\right)-f\left(x, u_{2}(x)\right)\right|=\lambda\left|u_{1}(x)-u_{2}(x)\right| \leq L \cdot\left|u_{1}(x)-u_{2}(x)\right| .
$$

If $\left|u_{1}-u_{2}\right|>0$ in a set of positive measure, we would find $\lambda \leq L$. Hence the solution of $(P)$ is unique.

Finally let us study the weak sequential continuity of $R_{\lambda}=\left(N_{f}-\lambda I d\right)^{-1}$ : $W^{1, p}(\Omega) \rightarrow W^{1, p}(\Omega)$. Suppose $R_{\lambda}(\psi)=u$, i.e., $f(x, u(x))-\lambda u(x)=\psi(x)$. Then

$$
\begin{aligned}
\|\psi\|_{W^{1, p}} & \geq \lambda\|u\|_{W^{1, p}}-\left\|N_{f}(u)\right\|_{W^{1, p}} \\
& \geq(\lambda-L)\|u\|_{W^{1, p}}-C .
\end{aligned}
$$

Writing in a better way, $\left\|R_{\lambda}(\psi)\right\|_{W^{1, p}} \leq \frac{\|\psi\|_{W^{1, p}}+C}{\lambda-L}$. We have just verified that $R_{\lambda}$ is a bounded operator. Suppose $\psi_{n} \rightarrow \psi$ in $W^{1, p}(\Omega)$. Let us set $u_{n}=R_{\lambda}\left(\psi_{n}\right)$. The sequence $\left\{u_{n}\right\}$ is bounded, therefore, up to a subsequence. We may assume that $u_{n} \rightarrow u$ in $W^{1, p}(\Omega)$. From the weak sequential continuity of $N_{f}-\lambda I d$, it follows that

$$
\psi_{n}=\left(N_{f}-\lambda I d\right)\left(u_{n}\right) \rightarrow\left(N_{f}-\lambda I d\right)(u) .
$$

This implies that $R_{\lambda}(\psi)=u$, and thus, $R_{\lambda}\left(\psi_{n}\right) \rightarrow R_{\lambda}(\psi)$ as desired.

Remark 5.2. Let us assume that $f(\cdot, t) \in W_{\text {loc }}^{1, \infty}(\Omega)$, and $\|f(\cdot, t)\|_{W^{1, \infty}(\tilde{\Omega})} \leq C(\tilde{\Omega})$, $\forall t \in \mathbb{R}$ and $\tilde{\Omega} \subset \subset \Omega$. Then the solution $u$ belongs to $W_{\text {loc }}^{1, \infty}(\Omega)$ provided the data $\psi$ lies in $W_{\text {loc }}^{1, \infty}(\Omega)$. In fact, for each $\tilde{\Omega} \subset \subset \Omega$, it follows that

$$
\|u\|_{W^{1, p}(\tilde{\Omega})} \leq|\tilde{\Omega}|^{1 / p} \cdot \frac{C(\tilde{\Omega})+\|\psi\|_{W^{1, \infty}(\tilde{\Omega})}}{\lambda-L}, \forall p>1 .
$$

Letting $p \rightarrow \infty$, we conclude the remark. Furthermore, this is as far as one can expect to go due to the obstruction imposed by the regularity of the nonlinearity $f$. 
The main information given by Theorem 5.1 is the regularization of the solution. We observe that if we see the map $\Lambda$ (defined in the proof of this theorem) as $\Lambda: L^{p}(\Omega) \rightarrow L^{p}(\Omega)$, it is easy to verify that it is a contraction. Therefore, from the Banach Fixed Point theorem, for all $\psi \in L^{p}(\Omega)$, the problem (5.1) has always a unique solution $u \in L^{p}(\Omega)$, provided $\lambda>L$. The main point of Theorem 5.1 is that $u \in W^{1, p}(\Omega)$ whenever $\psi \in W^{1, p}(\Omega)$.

Moreover the range provided by Theorem 5.1 is sharp, as the following simple example shows us:

Example 5.3. Let $f: \Omega \times \mathbb{R} \rightarrow \mathbb{R}$ be defined by $f(x, s)=|s|$. In this case, $\sup _{x \in \Omega}\|f(x, \cdot)\|_{\text {Lip }}=1$. Suppose $\lambda \leq 1$. Then $f(x, u(x))-\lambda u(x)=|u(x)|-\lambda u(x) \geq 0$. Hence, if $\psi \in W^{1, p}(\Omega)$, with $\psi(x)<0$, it is impossible to solve the equation (5.1).

\section{ACKNOWLEDGEMENT}

The authors would like to thank professor Haskell P. Rosenthal for his helpful suggestions. The authors are also grateful to Adam E. Parker for his careful revision throughout the elaboration of this article.

\section{REFERENCES}

1. R. Adams, Sobolev spaces, Academic Press, New York (1975). MR0450957 (56:9247)

2. H. Brezis, Analyse fonctionnelle, Théorie et applications. Collection Mathématiques Appliquées pour la Maîtrise. Masson, Paris, 1983. MR0697382 (85a:46001)

3. H. Brezis and E. Lieb, A relation between pointwise convergence of functions and convergence of functionals, Proc. Amer. Math. Soc. 88 (1983), 486-490. MR0699419 (84e:28003)

4. J. Diestel and J. J. Uhl, Jr., Vector measures, Mathematical surveys, No 15. AMS 1977. MR 0453964 (56:12216)

5. N. Dunford and J. Schwartz, Linear operator, Interscience Publishers, Inc., New York, second printing (1964). MR0117523 (22:8302)

6. R. Lucchetti and F. Patrone, On Nemytskii's operator and its application to the lower semicontinuity of integral functionals, Indiana University Mathematics Journal, Vol. 29 No 5 (1980). MR 0589437 (82i:47104)

7. H. P. Rosenthal, A characterization of Banach spaces containing $l^{1}$, Proc. Nat. Acad. Sci. U.S.A., 71 (1974). MR0358307 (50:10773)

8. M. Talagrand, Weak Cauchy sequence in $L^{1}(E)$, American Journal of Mathematics, 106 No 3 (1984). MF 0745148 (85j:46062)

9. T. Zolezzi, On Weak Convergence in $L^{\infty}$, Indiana Univesity Mathematics Journal, Vol. 23, No 8 (1974). MR0328576 (48"6918)

Department of Mathematics, University of Texas at Austin, RLM 12.128, Austin, TEXAs 78712-1082

E-mail address: dmoreira@math.utexas.edu

Department of Mathematics, University of Texas at Austin, Rlm 9.136, Austin, TEXAS 78712-1082

E-mail address: teixeira@math.utexas.edu 